\title{
Converting Wade-Giles Cataloging to Pinyin: The Development and Implementation of a Conversion Program for the Australian National CJK Service
}

\section{Linda Groom}

\begin{abstract}
In 1996, the National Library of Australia processed over 500,000 Chinese, Japanese, and Korean (CJK) USMARC records through a program that identified data in the Wade-Giles romanization scheme and converted it to the more user-friendly Pinyin romanization scheme. The conversion program was developed by the National Library in response to the needs of member libraries of the Australian National CJK Service. In this article, the author describes the development of the Pinyin conversion program, the functions of the program itself, and how it has been used in Australia. She also outlines the Pinyin word division standards adopted in Australia and briefly discusses the feasibility of an alternative methodology for the automatic creation of Pinyin data.
\end{abstract}

\footnotetext{
A

Lustralian libraries with collections of Chinese material have been faced, as have many libraries in other countries, with the problem of two competing romanization schemes for Chinese: Wade-Giles and Pinyin (Lu 1995; Tao and Cole 1990; Young 1992). In essence, the problem for Australian libraries has been that, while users of Australian Chinese collections generally have a strong preference for Pinyin romanization, most of the Chinese data in Australian library catalogs are in Wade-Giles. For some library users, especially users in public
}

libraries and undergraduates learning Chinese, Wade-Giles romanization is almost incomprehensible. The cost of manually converting library catalogs from Wade-Giles to Pinyin has, however, been prohibitive.

In June 1996, the Australian National CJK Service was established. The CJK Service is an online service maintained by the National Library of Australia. It provides bibliographic data for copy cataloging and location information for CJK materials held in Australian libraries. Around $90 \%$ of the bibliographic records 
contain the original ideographic characters, as well as romanization.

During the three-year project to establish the CJK Service, the National Library took the opportunity to develop a conversion program that automatically converts Wade-Giles bibliographic records to Pinyin. This program has been used to process over 500,000 bibliographic records, including all the $\mathrm{CJK}$ records available through the Library of Congress Cataloging Distribution Service, and selected records from the OCLC Online Computer Library Center, Inc., and Research Libraries Information Network (RLIN) databases. The conversion program allowed the creation of parallel Pinyin and Wade-Giles databases, each containing CJK records and ideographic characters, but using different Chinese romanizations.

\section{The Decision to Create a National Pinyin Database for Australia}

Through the late 1980s, Australian libraries debated with some vigor the merits of the two romanization schemes, notably at the annual user meetings of the Australian Bibliographic Network (ABN). In response to this, the National Library of Australia commissioned a study by MacDougall (1991). After considering MacDougall's study and comments from Australian libraries, the ABN Standards Committee felt there was no option but to allow both romanization schemes on the ABN database.

In 1995, as the implementation date of the National CJK Service database drew nearer, the debate was rekindled. The forums for the debate were the meetings of the CJK Technical Committee, the committee charged with developing cataloging policy for the National CJK Service. Chaired by Chooi Hon Ho of Monash University, the committee comprised representatives from each of the initial eight member libraries of the CJK Service.

At the Technical Committee's meeting in Canberra on February 14 and 15, 1995, much time was spent on the question of which scheme should be adopted for the romanization of Chinese in original cataloging on the National CJK Service database. As might be expected, the committee members representing the libraries with the largest and oldest Chinese collections and with large catalogs in WadeGiles, such as the National Library itself and the Australian National University, argued in favor of adopting Wade-Giles as the National CJK Service standard. Committee members representing libraries with more recently developed collections argued in favor of Pinyin. The issue was not resolved at the meeting.

The member libraries were, however, unanimous on one point: that Australian libraries should do everything possible to move to a single standard for the romanization of Chinese. There was a strong view that the dual romanization policy that had been used for $\mathrm{ABN}$ cataloging was not working. The inefficiencies of having two standards, and the failed searches that resulted when these two standards occurred in either a national shared database or a library's own catalog, were clear and urgent problems.

It was with the knowledge that a solution was needed that I attended the First International Seminar on Chinese Document Database, in Guangzhou, February 27, 1995, to March 4, 1995. There I had the good fortune to meet Karl Lo, International Relations and Pacific Studies Librarian at the University of California, San Diego, and author of a program that converted Wade-Giles words to Pinyin (Lo and Miller 1991). The program was designed to work with input strings that were guaranteed to be in Wade-Giles, converting these strings letter by letter. Machine-Readable Cataloging (MARC) records for Chinese material in Western library catalogs contain a great deal more than Wade-Giles-for instance, alphabetic subfield codes and notes and subject headings in English. I had unpleasant visions of what such a program might do to phrases such as "edited by" or "title page."

I posed this problem to Lo during the conference. Was it feasible for a program to distinguish Wade-Giles words from all other words in a MARC record and to convert those words, and only those 
words, to Pinyin? In Lo's view, it was entirely feasible. He responded to every concern raised with an indication of what could be accomplished in machine conversion by this program, and in the end I was convinced. He has since told me that if he had seen some of our actual data, he would have been far less optimistic. Still, I came away from the conference inspired. I am tempted to wonder how many other projects have had origins that include an element of frank and friendly international misunderstanding!

In March, a small team at the National Library began work on creating a program that would read USMARC records, identify any Wade-Giles words in them, convert the Wade-Giles to Pinyin, and reassemble a valid USMARC record. The team consisted of two National Library staff: Ching-Ping (CP) Tang and Murthy Manchella, with Karl Lo as consultant. We experimented with letter-by-letter conversion and word-byword conversion and chose the latter. By May, we had a working program and were able to produce sample data acceptable to the CJK Technical Committee. Two months later, the CJK Steering Committee approved the establishment of a Pinyin database that would be a mirror image of the Wade-Giles database.

\section{Description of THE WADE-Giles to Pinyin Conversion Program}

The program is written in C. It accepts files of USMARC records, in batches of up to 20,000 records. It first checks that the MARC record contains the 001 (record number) field, since the program needs this field to keep track of each record. It then reads the language code in the 008 field, if present, and divides the file into Chinese, Japanese, Korean, and other. This division by language is necessary because later steps in the program use different logic for records whose main language is not Chinese.

Readers of this article may wonder why we bothered at all with MARC records for Japanese, Korean, and other material. We needed to put all records through the conversion to catch, for example, Japanese translations of Chinese works, where the author name and uniform title were in Wade-Giles. This requirement added considerably to the complexity of the program.

\section{MARC TAG TABLE}

After dividing the file by language, the program processes each MARC record in turn. For each record, it consults a table that lists all the USMARC fields and subfields that, in the team's view, are likely ever to contain a Wade-Giles word. Some fields, such as 008 and 040 , which contain codes or symbols, should never contain any Wade-Giles words and so can be safely excluded from the conversion process. The table of MARC fields and subfields grew with each version of the program, as Wade-Giles data turned up in unexpected places. For example, field 020 subfield a (ISBN), which one might expect to be totally numeric, sometimes contains a Chinese publisher name. It might have been simpler in the end to list only those fields in which Wade-Giles would not occur. It would have been a much shorter list.

\section{Main Processes}

Each word in each subfield is then put through a sequence of tests, which aim to determine whether the subfield contains Wade-Giles or not. If the subfield contains Wade-Giles and only Wade-Giles, it is converted. If it contains no Wade-Giles, it is left untouched. If the program cannot decide whether the subfield contains Wade-Giles, the program converts it and flags the preconversion image and the postconversion image for human review.

To make the sequence of tests, the program refers to the "word table" that lists all valid Wade-Giles words. The following attributes are specified for each Wade-Giles word:

- Its Pinyin equivalent.

- Whether its Pinyin equivalent is the same. For example, the Wade-Giles word "ang" converts to Pinyin "ang"; these words are called "same" words.

- Whether there is a Pinyin word that contains the same letters, but has a different meaning. For example, "chao" is valid in both Wade-Giles and 
Pinyin, but Wade-Giles "chao" converts to Pinyin "zhao." These words are referred to as "common" words.

- Whether the Wade-Giles word also happens to be valid in Hepburn (Japanese romanization) or McCune-Reischauer (Korean romanization).

The tests use a process of elimination. The broad structure of the tests is listed below.

1. Personal name processing. If a personal name field contains a non-Wade-Giles word, e.g., "Jimmy Chang" where "Jimmy" is non-Wade-Giles, and "Chiang Kai-shek" where "shek" is non-WadeGiles, the program leaves the entire name unconverted.

2. Pure Wade-Giles processing. If a subfield contains only pure Wade-Giles words-i.e., words that are unique to Wade-Giles-it is converted. The program also tests for subfields that contain other combinations involving pure Wade-Giles words. For example, subfields that contain both a pure Wade-Giles word and a pure Pinyin word are flagged for human review. Subfields that contain a pure WadeGiles word and an English word (the program categorizes English words as "nontable" words) are also flagged for human review, unless they are in fields such as subject (6xx) fields. Subject fields often contain phrases like "T"ang dynasty," where the presence of the English word does not cast doubt on the fact that " $T$ ' ang" is Wade-Giles. In these cases, the pure Wade-Giles word is converted, and the phrase becomes "Tang dynasty."

3. Pure Pinyin processing. We found it necessary to put in a test aimed at weeding out Pinyin data, because databases that are supposedly WadeGiles contain a surprising amount of Pinyin. If the subfield consists entirely of pure Pinyin words, then it is not converted. The program also tests for subfields that contain other combinations involving pure Pinyin words, for example, a pure Pinyin word and some "common" words or a pure Pinyin word and an English word. Because of the frequency of
English words in statements of responsibility-e.g., "translated by Wen Zhong"-special processing occurs for statements of responsibility.

4. Processing of non-Chinese words. If the subfield consists entirely of words that are not listed in the word table (i.e., none of the words are WadeGiles), then it is not converted. The program also tests for subfields that contain other types of words, such as nontable words plus "same" or "common" words. Fields such as general notes $(500)$ or chronological subfields in subject headings (6xx subfield $y$ ), where English words are often mixed with Wade-Giles words, are flagged for human review. Human review seems the only reliable way to ensure that notes data, such as "Running title Shang-hai kuan pao," are correctly converted to "Running title Shanghai guan bao."

5. "Common" words processing. If the subfield contains any words that occur in both Wade-Giles and Pinyin but with different meanings, and if the subfield is in a field such as a title or note field, it is flagged for human review. Other subfields containing "common" words are converted.

6. "Same" words processing. If the subfield contains any words that occur in both Wade-Giles and Pinyin and have the same meaning, it is converted. Since these words are the same in Wade-Giles and Pinyin, conversion does not appear to take place, except where two Wade-Giles words are hyphenated. Thus "Yang-wen" converts to "Yangwen."

7. Remaining subfields are not converted.

\section{Processing of The Wade-Giles WORDS “Tr" AND "Lo"}

Most Wade-Giles words have a one-toone conversion to Pinyin. The Wade-Giles words "ti" and "lo," however, each have more than one Pinyin equivalent. The word "ti" can convert to either "di" or "de," and "lo" can convert to either "le" or "luo." To make the correct conversion, the program searches the linked 880 field to 
find the Chinese character that is linked to the "ti" or "lo." From the code for that character, the program can correctly convert the word. If the record does not contain any Chinese characters, the program checks the field and subfield in which the word occurs. $A$ " $t$ " occurring in a personal name (100 \$a, $600 \$ \mathrm{a}$, etc.), for instance, can be converted to "di" with a high degree of reliability. In some subfields, however, the correct conversion cannot be predicted; these are flagged for human review.

\section{Human Review of Doubtful Cases}

Each batch of records produces some cases that are flagged for human review. These files can be transferred to the reviewer's computer using file transfer protocol (FTP) and reviewed using a commercial editing package such as Microsoft Write or reviewed on the machine on which the conversion program resides, using GNU Emacs, a UNIX-based editor.

The human reviewers are presented with the field before conversion, the field after conversion, and a brief message saying why the field has been flagged for review. The reviewer does not see the entire bibliographic record. This produced some disadvantages, in that human reviewers had to open another window to the CJK Service database to check a romanized field against the Chinese characters carried in the linked 880 field. A design that involved processing entire MARC records, however, would have produced slow response times both in calling up files and in other processing.

Reviewers choose to keep either the unconverted or the converted version. They can also edit the converted version of the field if they wish.

\section{Final Processing}

The reviewed fields are then merged back with the other fields in the postconversion bibliographic record. The program checks to see whether there is a title added entry (740) that, after conversion, is now the same as the main title (245). If it is exactly the same, the 740 field is deleted. The program finally recalculates the MARC directory and reassembles a valid USMARC record.

\section{WORD DIVISION STANDARdS}

In preparing for the development of the Pinyin database, the CJK Technical Committee faced a situation in which there was no internationally recognized standard for Pinyin word division. In 1995, the committee decided to set the word division standards for use by catalogers using the National CJK Service (1996, resolution 5) stating:

That where a cataloger inputs Pinyin data into the National CJK System, each Chinese character should be input as one Pinyin syllable, except for proper and geographic names, where the syllables should be joined: Thus the Wade-Giles Mao Tse-tung is converted as Mao Zedong.

Considerable discussion preceded this decision. Pinyin word division standards have been a controversial topic for many years in Australia. The Technical Committee considered a range of issues, including the effects of single-syllable Pinyin on keyword searching, the extent to which proximity searching could help, the need for an authoritative dictionary if the joined-syllable approach was adopted, and the emerging practice of the National Library of China, in which recent MARC record samples contained single-syllable Pinyin. Another aspect that influenced the Technical Committee was the Committee's enthusiasm for "automatic romanization," a prospective enhancement to the Innopac software under which catalogers would enter Chinese characters only, and Innopac would automatically create the parallel romanized fields. Automatic romanization is easier to implement if each character is romanized as a single syllable. Perhaps the crucial factors were that, although the Committee recognized that there were good arguments on both sides, they felt that a decision was better than further years of debate, and that for practical reasons a standard was needed that was simple and easy for catalogers to apply. The CJK Technical Committee also decided (1996, resolution 12) that the 
Record source: $\mathrm{ABN}$

Before conversion: $245 \$$ a Ch'un ts'un

After conversion: 245 \$a Ch'un ts'un

Figure 1. Unexpected Diacritics.

policy for authorities on the Pinyin database should be as follows:

That ... in the Pinyin database:

- where the preferred form in LCNA is a pure Wade-Giles form, the preferred form in the Pinyin database should be that Wade-Giles form converted into Pinyin

- where the preferred form in LCNA is an "established" i.e. not a pure Wade-Giles form, for instance Chang Kai-shek and Confucius, the preferred form on the Pinyin database should be that "established" form.

The word division standard has since been proposed by the Library of Congress for adoption by American libraries (Melzer 1996).

\section{CONVERSION ERrors}

The National CJK Service Pinyin database contains some incorrect data. Although we have now corrected the causes of many of these errors in the program, we have not been able to correct all of the records on the database that contain errors. Some examples of error types are listed below:

1. Unexpected diacritics. The diacritics in "ch'un" and "ts'un" in figure 1 had been incorrectly keyed as alifs; they should have been keyed as ayns. The presence of the alifs meant that the conversion program did not recognize "ch-n" and "ts-n" as Wade-Giles words and left them unconverted. (Note that the conversion program does predict the common cataloger shortcut of typing apostrophes instead of ayns and makes correct conversions. We had not predicted the possibility of a cataloger typing an alif instead of an ayn.)

2. Unexpected tag and subfield combinations. The "chih shih" in figure 2 was not converted because we had not listed $710 \$ \mathrm{~d}$ in the table of data elements to be processed. It has now been listed in the table.

3. Unexpected subfield coding. The $\$ \mathrm{~h}$ subfield in figure 3 was incorrectly left in Wade-Giles. The \$h subfield should have been coded as a $\$ b$; $\$ \mathrm{~h}$ was not listed in the MARC tag table. We have now added $245 \$$ h to the MARC tag table, to catch cases such as this in future.

4. Records with Chinese titles but with language code "jpn." Although the uniform title field (130) in figure 4 was converted correctly, the main

Record source: LC (90216819)

Before conversion:

710 \$a Tseng, Tso-chou, \$d chih shih 1844

After conversion:

710 \$a Zeng, Zuozhou, \$d chih shih 1844

Figure 2. Unexpected Tag and Subfield Combinations. 
Record source: LC (94452207)

Before conversion:

$245 \$ a$ Ch_n shan chih shang: \$h [sin ch ao san wen hs_an ts ui] / \$c Li Fu-wei...

After conversion:

$245 \$ a$ Qun shan zhi shang : \$h [sin ch ao san wen hs_an ts ui] / \$c Li Fuwei...

Figure 3. Unexpected Subfield Coding.

title (245) was not converted. The program does not process 245 fields when the language code is "jpn." If the record had been correctly coded as "chi" instead of "jpn," the conversion would have occurred without error. The program could be amended to process the 245 fields even when the language code is "jpn," but this would cause a large number of fields to be flagged unnecessarily for human review. Incorrect language coding is one of the most common sources of conversion errors.

5. Japanese records with parallel titles in Chinese. In figure 5 , the language coding was correct. However, for records with language code "jpn," we had not listed the $245 \$ \mathrm{~b}$ subfield for processing. If $245 \$ \mathrm{~b}$ was listed, many subtitles in Japanese that contained words also valid in WadeGiles would be needlessly flagged for human review. However, the result of not listing $245 \$ b$ for jpn records is that Chinese parallel titles in Japanese records are skipped by the con- version. This was a pragmatic decision to save reviewing time. The program could be easily amended to check $245 \$$ b's in Japanese records.

6. Diacritics omitted. "Tien-chin" in figure 6 was incorrectly converted to "Dianjin," and "yu" was incorrectly converted to "you," because the cataloger omitted diacritics.

7. Some notes become incongruous. Some Wade-Giles records contain a note such as "Added title also in Pinyin. ..." Once the record has been converted to Pinyin, such notes are incongruous. Although it would be possible to write software to detect and delete many of these notes, we decided it was not worth the effort.

It is tempting to dismiss many of the above problems as resulting from cataloger error in the original data and to say that the conversion program is not at fault. Catalogers are only human, and errors are part of life, however. Our policy has been to code in expectation of cataloger errors where possible. In creating the Pinyin database, the reviewers

Record source: OCLC

Before conversion:

$008 \ldots . j p n$

$130 \$ a$ Ch'ang-chiang jih pao.

$245 \$ a$ Tu pao shou ts'e, \$c Ch'ang-chiang jih pao pien.

After conversion:

$008 \ldots . . j p n$

$130 \$ a$ Chang jiang ri bao

245 \$a Tu pao shou ts e, \$c Ch'ang-chiang jih pao pien.

Figure 4. Records with Chinese Titles but with Language Code "jpn." 
Record source: LC (91229795)

Before conversion:

$008 \ldots . . j p n$

245 \$a Shinpoj umu Nitch u Jid o Bungaku no Kin o

Ky o soshite Asu h okokusho : \$b 1990-nen 10-gatsu 13-nich-15-

nichi $=\mathrm{Hs}$ _eh shu $\mathrm{t}$ ao lun hui Chung-Jih erh $\mathrm{t}$ ung wen

hs_eh ti kuo ch _ hsien tsai chi ch i chiang lai

$t$ sai liao hui pien

After conversion, there was no change.

Figure 5. Japanese Records with Parallel Titles in Chinese.

also picked up a number of cataloger errors and corrected them as part of the reviewing process.

\section{Management Problems}

A large problem in management of a conversion using this program is the provision of staff to review the data flagged as doubtful conversions. At present, the Wade-Giles to Pinyin program flags around $12 \%$ of all fields for review. For the conversion of the National CJK Service database of over 500,000 bibliographic records, a total of 920 hours of human reviewing, not including supervisory time, over a period of two months was required. It would be easy to change the program's logic so that fewer data were flagged for human review, but this would produce more conversion errors.

Another major management problem for the National Library of Australia has been the workload and complexities of maintaining parallel Wade-Giles and Pinyin databases. With the help of the software supplier Innovative Interfaces, we have solved a number of the problems of cross-updating the two databases, but some problems remain.

\section{How the Conversion Program Has Been Used by the National CJK SERvice}

Libraries that wish to convert to Pinyin can use the Pinyin database as a migration tool. For the many libraries that added holdings to the $\mathrm{ABN}$ for their CJK material, the migration path is relatively smooth. Their holdings have been copied to the Pinyin database, allowing those libraries easily to extract a Pinyin version of MARC records for all the CJK material they own. These MARC records can then be loaded to the library's local system, where they replace Wade-Giles records. A number of libraries are planning to use the Pinyin database in this way. Libraries whose holdings are not on the CJK Service database will need to add their holdings to it retrospectively in order to be able to extract the Pinyin records. Alternatively, they could perform a local conversion to Pinyin using the Wade-Giles to Pinyin conversion program directly on their local data. The

Record source: ABN (interim record)

Before conversion:

260 \$a Tien-chin: Tien-chin chiao yu chu pan she, 1989

After conversion:

260 \$a Dianjin: Dianjin jiao you shu ban she, 1989.

Figure 6. Diacritics Omitted. 
National Library will make the conversion program available to any CJK Service member libraries that wish to use it.

The CJK Technical and Steering Committees have agreed that the existence of the Wade-Giles database will be reviewed in mid-1998. At that point, it will be decided whether the workload of supporting two databases is worthwhile. The ultimate aim is to meet the member libraries' requirement for the adoption of a single Chinese romanization scheme in Australia; given the trend among the member libraries, it seems almost certain that the single romanization scheme will be Pinyin. A likely option thus seems to be to cease support for the WadeGiles database; the timing of that would, however, depend on the extent to which member libraries are able to migrate their local systems to Pinyin.

\section{Other Methodologies}

Some readers of this article may wish to question the wisdom of putting such programming effort into romanization. Romanization is, after all, an extremely poor substitute for the original characters. For many Australian libraries with Chinese collections, however, it will be some years until they acquire local systems that can support Chinese characters. These libraries need a source of MARC data that will allow them to present their cataloging in the romanization scheme most familiar to their users. Even in universities where the library may have installed a library system that supports CJK characters, library users often connect to the library catalog from their departments and offices, and few of these users yet have the software on their desktop PCs to display CJK characters. My personal view is that the debate over whether to use Wade-Giles or Pinyin has consumed librarians' energies too long; by moving to a single standard, librarians can focus on the more productive task of presenting cataloging data in the original script.

A second argument against expending effort on converting one romanization scheme to another is that it would be better to store the data in its original character form only and to build software that will create a view "on the fly" in whatever romanization scheme the user prefers. This approach is very attractive. It puts the emphasis where it should be-on the original characters. It covers all user preferences by offering choices. It would allow any new romanization schemes to be supported by simply adding another table to the software that creates the "views." It would allow catalogers to create records containing only the original characters, without the double work of having to create romanization. It would also allow wider use of the MARC records created in countries such as China and Korea; such MARC records normally do not contain any romanization.

On the other hand, there are a number of issues that need to be faced before libraries can create records containing only the original characters and rely on software to meet all romanization requirements. Some of these issues are listed below.

- All the catalog data for non-Roman material would need to contain the original characters; retrospective conversions to add original characters will need to be done by some libraries.

- Many users will still wish to use romanization in search terms, in cases where they find it quicker to type a romanized word than to create an ideographic character. In some cases, where a Chinese character with the same appearance has more than one equivalent code, searches on romanized terms produce more reliable results. To meet this need for romanized searching, library systems that stored only the original characters would need to develop the ability to search a romanized search term against indexes that contained the original characters.

- Although some library systems have developed software that will convert Wade-Giles search terms to Pinyin (such as OCLC) or assist catalogers by creating romanization from ideographic characters (such as Innopac, Dynix), there are no library systems, to my knowledge, that yet offer or plan to offer the full views capability.

- Decisions would need to be made on what to do with the romanized data 
that presently exist, along with original character data, in MARC records; should they be discarded?

- Cataloging issues-such as when is a view of a heading a view, and when is it an unpreferred form? - would need to be addressed.

- The USMARC format, which enshrines romanization in the main fields ( $1 \mathrm{xx}, 245$, etc.) might need to be changed, with consequent changes to library systems.

\section{Conclusron}

The conversion of the 500,000 -record National CJK Service database has demonstrated that it is possible to convert bibliographic data from Wade-Giles to Pinyin with a high degree of automation of the conversion process. It has also demonstrated that the libraries of one Western nation, at least, can reach the agreement on Pinyin word division standards that is necessary for a large conversion project.

Some problems remain-including the conversion of authority data and the conversion of general catalogs where Wade-Giles data are scattered among many Western languages. Although it would be foolish to underestimate these remaining problems, Australian libraries now have a clear path toward their vision of a single Chinese romanization scheme, and toward its consequent benefits of more reliable searching for users, and greater opportunities for exchange of Chinese cataloging data.

\section{WORKS CITED}

Lo, Karl K., and R. Bruce Miller. 1991. Computers and romanization of Chinese biblioraphic records. Information technology \& libraries 10: 221-33.

Lu, Suping. 1995. A study on the Chinese romanization standards in libraries. Cataloging \& classification quarterly 21, no. 1: $81-96$.

MacDougall, Susan. 1991. Parallel Chinese romanization on the Australian Bibliographic Network. Canberra: [National Library of Australia].

Melzer, Philip. 1996. Pinyin romanization: Word division recommendation. Chinese librarianship: An international electronic journal 2 (Dec. 1): http://www.lib.siu.edu/ swen/iclc/clej.htm.

National CJK Service. Technical committee. 1996. List of resolutions. March 11. National Library of Australia, Canberra.

Tao, Hanyu, and Charles Cole. 1990. WadeGiles or Hanyu Pinyin: Practical issues in the transliteration of Chinese titles and proper names. Cataloging \& classification quarterly 12, no. 2: 105-17.

Young, Joann S. 1992. Chinese romanization change: A study on user preference. Cataloging $b$ classification quarterly 15, no. 2: $15-28$. 